\title{
Improved synthetic TP-AGB models
}

\author{
P. Marigo \\ Max-Planck-Institut für Astrophysik, Garching bei München, Germany
}

\begin{abstract}
We present recent improvements and results of an extensive analysis of the TP-AGB phase performed by means of a synthetic model (Marigo 1998a, b; Marigo et al. 1998a, b). The improvements concern: i) the use of a homogeneous and accurate set of analytical relations (Wagenhuber \& Groenewegen 1998); ii) a new treatment of envelope burning in the most massive TP-AGB stars $\left(M>3.5 M_{\odot}\right)$ to account for the possible break-down of the core mass-luminosity relation; iii) a better treatment of the third dredge-up to infer if and when the process takes place.

Extensive calculations of synthetic TP-AGB models have been carried out over the mass range $\left(0.8 M_{\odot} \div 5 M_{\odot}\right)$ and for three sets of initial metallicity $(Z=0.019, Z=0.008, Z=0.004)$. The formation of carbon stars is investigated addressing the following issues: a) the reproduction of the observed luminosity functions of carbons stars in both Magellanic Clouds, and b) the formation of very bright and optically obscured carbon stars.
\end{abstract}

\section{Introduction}

Synthetic TP-AGB models may be a powerful tool of investigation, thanks to quick computing and easy testing of different prescriptions. These characteristics make them complementary to complete but extremely time-consuming calculations of stellar models. To this respect, we report here on recent effort made to improve the degree of accuracy of the synthetic approach, and discuss some applications.

\section{A consistent treatment of envelope burning}

Synthetic TP-AGB calculations have so far adopted the erroneous assumption that the core mass-luminosity $\left(M_{\mathrm{c}}-L\right)$ relation determines the surface luminosity even of stars experiencing efficient envelope burning (or hot-bottom burning; e.g. Renzini \& Voli 1981; Groenewegen \& de Jong 1993; Marigo et al. 1996). Marigo et al. (1998a) and Marigo (1998ab) have made the first effort to overcome this inadequacy, by developing a solution scheme, based on envelope integrations, in which the equation of energy balance is included and integrated with the aid of proper boundary conditions. The method turns out to be reliable as it reproduces with great accuracy the results of full calculations (i.e. Blöcker \& Schönberner 1991; Blöcker 1995; see Fig. 1). 

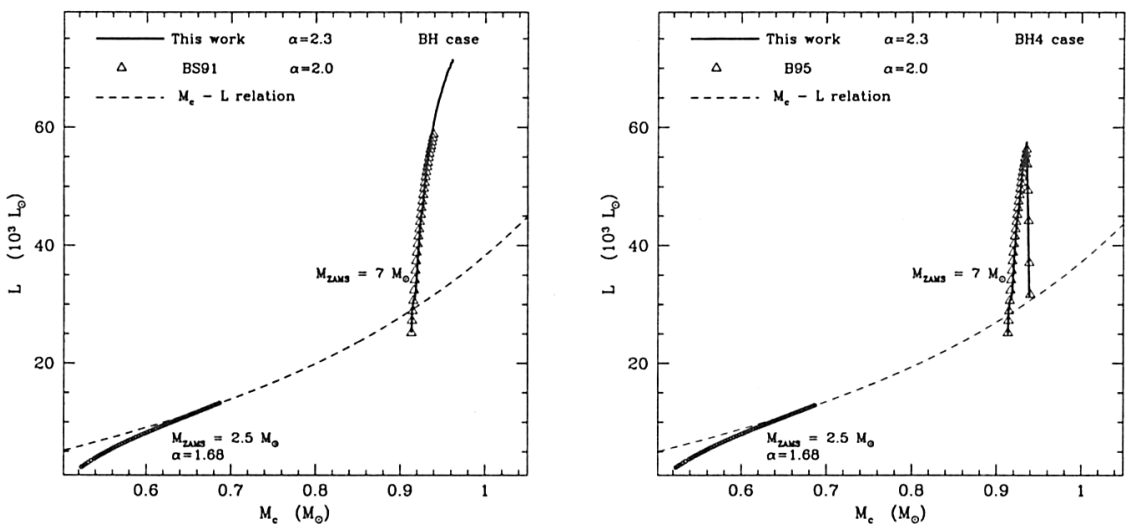

Figure 1. Quiescent luminosity evolution as a function of the core mass for a $7 M_{\odot}$ TP-AGB model of solar composition, according to two prescriptions for mass loss: 1) Baud \& Habing 1983, (BH case, left panel); 2) the same as the former case up to the activation of a constant mass-loss rate of $10^{-4} M_{\odot} \mathrm{yr}^{-1}$, (BH4 case, right panel). The predictions of full calculations are denoted by triangles [Blöcker \& Schönberner 1991 (BS91); and Blöcker 1995 (B95)] at the stage of the pre-flash luminosity maximum. The results of the present synthetic calculations are shown (solid line), starting from the same initial conditions at the first thermal pulse. See Marigo (1998b) for more details.

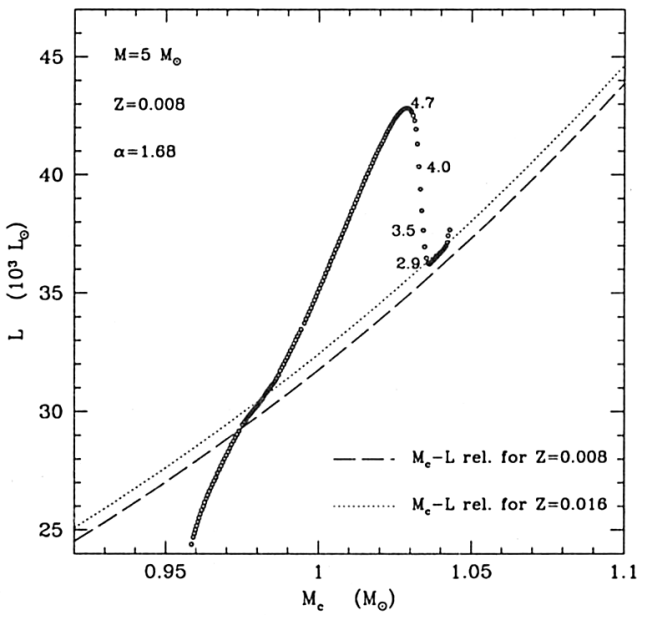

Figure 2. Quiescent luminosity evolution as a function of the core mass for a $5 M_{\odot}, Z=0.008$ model experiencing envelope burning. A few values of the current stellar mass are indicated in $M_{\odot}$. The massloss prescription is according to Vassiliadis \& Wood (1993). 

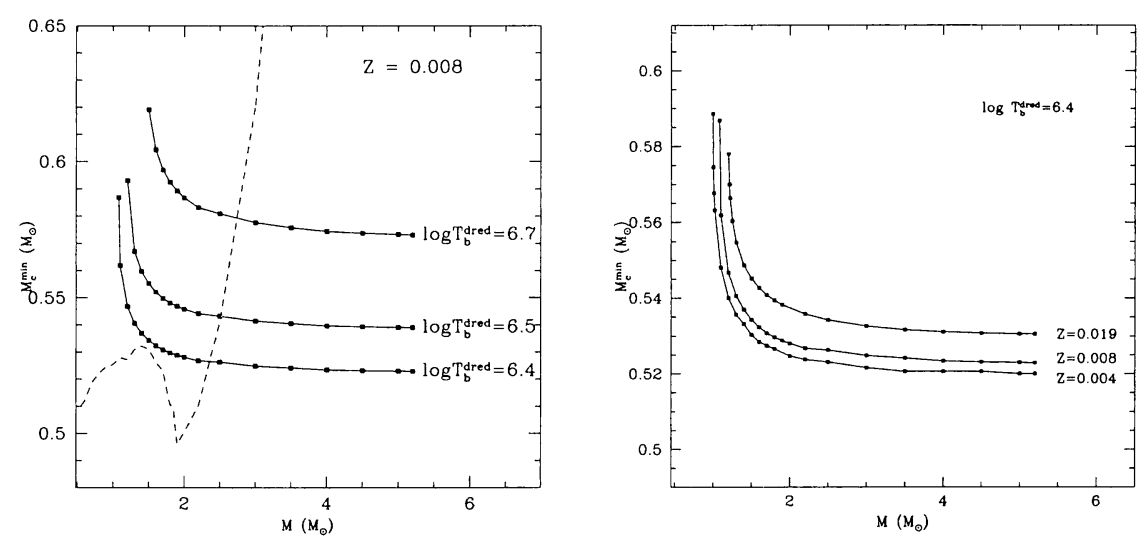

Figure 3. Minimum core mass $M_{\mathrm{c}}^{\text {min }}$ derived from envelope integrations as a function of stellar mass and metallicity, for different choices of the temperature parameter $T_{\mathrm{b}}^{\text {dred }}$. The core mass at the first thermal pulse, for models with initial metallicity $Z=0.008$, is shown (left panel; dashed line).

The typical evolution of the quiescent luminosity, as a function of the core mass, described by a TP-AGB star with envelope burning is shown in Fig. 2, which refers to a $\left(5 M_{\odot}, Z=0.008\right)$ model. The luminosity first increases above the $M_{\mathrm{c}}-L$ relation, reaches a maximum at the onset of the superwind regime, then quickly drops as envelope burning weakens because of the significant reduction of the envelope mass, and eventually approaches the $M_{\mathrm{c}}-L$ relation, which is followed till the end of the evolution. Note that the final recovering of the $M_{\mathrm{c}}-L$ relation is consistent with a current envelope metallicity nearly twice larger $(Z=0.016)$ than the original one $(Z=0.008)$, due to the effect of both dredge-up and envelope burning.

\section{The third dredge-up: if and when from envelope integrations}

The usual treatment of the third dredge-up in synthetic TP-AGB models is based in the use of two free parameters, namely: the minimum core mass $M_{\mathrm{c}}^{\text {min }}$ for convective dredge-up to occur; and the efficiency $\lambda=\Delta M_{\mathrm{dred}} / \Delta M_{\mathrm{c}}$, i.e. the fraction of the core mass increment over an inter-pulse period which is dredgedup to the surface at the subsequent thermal pulse.

For the sake of simplicity, both parameters have been often assumed constant in synthetic calculations, regardless of the stellar metallicity and mass (e.g. Groenewegen \& de Jong 1993; Marigo et al. 1996). On the contrary, full modelling of the third dredge-up shows that their properties vary according to many factors, i.e. treatment of convection, core and envelope mass, metallicity, pulse strength, numerical details (see, for instance, Mowlavi, in this volume).

In this context, the synthetic treatment of the third dredge-up is improved as briefly described. According to the indications from complete calculations 


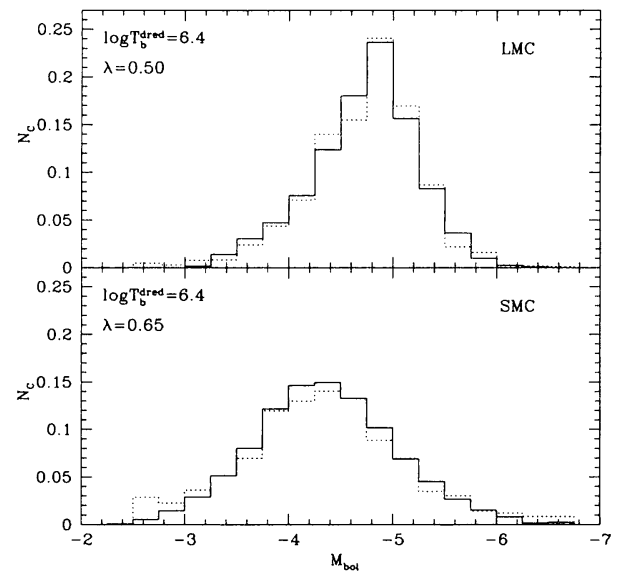

Figure 4. Carbon star luminosity functions in the Large and Small Magellanic Clouds (LMC and SMC). Observations (dotted line) are those by Costa \& Frogel (1996) for the LMC, and Rebeirot (1993) for the SMC. Theoretical best fits (solid line) are obtained adopting the dredge-up parameters as indicated, and a constant star formation rate history. See Marigo et al. (1998b) for more details.

of thermal pulses (Wood 1981; Boothroyd \& Sackmann 1988), the occurrence of the third dredge-up requires that the base of the convective envelope attains a minimum temperature, $T_{\mathrm{b}}^{\text {dred }}$, at the stage of the post-flash luminosity maximum. Then, we relax the usual criterion based on a constant $M_{\mathrm{c}}^{\mathrm{min}}$, and replace it with one on the base temperature to infer if and when dredge-up takes place (see also Wood 1981). To this aim, envelope integrations are performed, in which the post-flash luminosity maximum is given by the core mass-peak luminosity relation by Wagenhuber \& Groenewegen (1998). In this way, we are able to predict both the onset and the shut-down of the third dredge-up as a function of the stellar mass $M$, metallicity $Z$, and pulse strength.

The behaviour of $M_{\mathrm{c}}^{\min }(M, Z)$, as derived from envelope integrations, is displayed in Fig. 3 for different choices of the temperature parameter $T_{b}^{\text {dred }}$. All the curves share a common trend, i.e. $M_{\mathrm{c}}^{\min }$ steeply increases at lower masses, and flattens out to a nearly constant value towards higher masses. It is worth noticing that the onset of dredge-up is favoured at lower metallicities, in agreement with the results from complete calculations.

\section{The carbon star luminosity functions in the Magellanic Clouds}

The calibration of both dredge-up parameters is carried out in order to fulfil the constraint given by the observed luminosity functions of carbons stars in the Magellanic Clouds (see Marigo et al. 1998b). It turns out that $T_{\mathrm{b}}^{\text {dred }}$ essentially controls the faint end of the distribution (together with the effect due to the longlived luminosity dip induced by thermal pulses in lower mass TP-AGB stars). Once the proper value of $T_{\mathrm{b}}^{\text {dred }}$ is singled out, the efficiency parameter is changed 


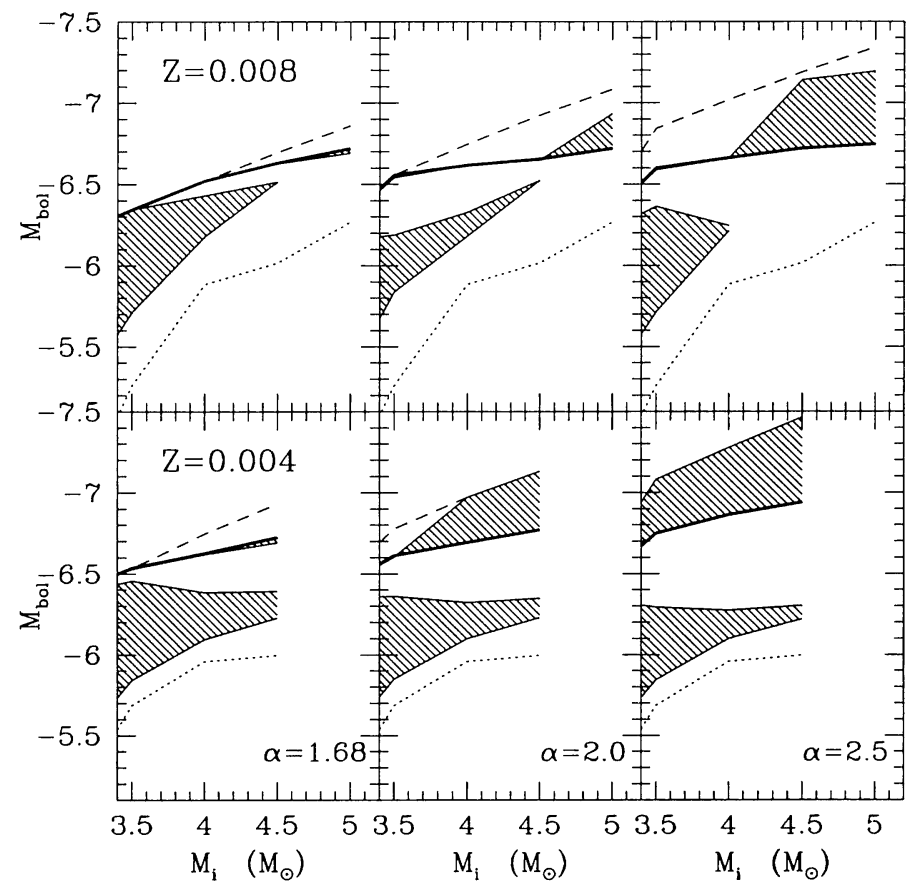

Figure 5. Transition luminosities as a function of the initial mass defining: the onset of the TP-AGB phase (dotted line), the carbon star domain (shaded areas), the AGB termination (thick solid line), the maximum luminosity attained during the TP-AGB evolution (dashed line). Each frame is characterised by the pair $(Z, \alpha)$. See the text for more details.

so as to reproduce the location of the peak of the distribution. Indeed, both features (i.e. the faint wing and the peak) are strong calibrators of the dredgeup parameters, whereas other properties of stellar populations (i.e. history of the star formation rate; initial mass function) are found to play a minor role.

It is interesting to notice that the best fits, displayed in Fig. 4, are obtained adopting the same value of the temperature parameter $\left(\log T_{\mathrm{b}}^{\mathrm{dred}}=6.4\right)$, and invoking a larger efficiency of dredge-up for the SMC $(\lambda=0.65)$ than for the LMC $(\lambda=0.50)$. Both findings are in a qualitative agreement with the expectations from full calculations of the third dredge-up, i.e. $T_{\mathrm{b}}^{\text {dred }}$ is constant and dredge-up is favoured at lower metallicities.

\section{The formation of massive and luminous carbon stars}

The improved treatments of envelope burning and dredge-up allow to investigate the formation of high-luminosity carbon stars (Marigo, in preparation). More 
massive TP-AGB stars $\left(M>3.5 M_{\odot}\right)$ can make the transition to the carbon star domain as the third dredge-up dominates over envelope burning in the final part of their evolution. These stages are characterised by quite high values of the luminosity (due to the over-luminosity effect produced by envelope burning), and heavy mass-loss rates (after the onset of the super-wind) (cf. Frost et al. 1998; see also Lattanzio \& Forestini, this volume).

Fig. 5 shows the predicted luminosity range spanned by carbon stars, for initial masses in the range $3.5 \div 5.0 M_{\odot}$, as a function of metallicity $Z$, and mixinglength parameter $\alpha$. These models can experience one and/or two transition(s) to the the carbon star domain. The earliest one occurs as envelope burning is growing in efficiency, but still weaker than the third dredge-up. Indeed, this first transition can be even prevented by the occurrence of envelope burning. The latest transition usually takes place in the final stages when envelope burning is weakening and the third dredge-up becomes dominant again.

However, this standard picture may break down in TP-AGB models in which envelope burning is so efficient (e.g. for high $\alpha$ and low $Z$ ) and operating for a time long enough to activate the ON cycle. Under these circumstances, envelope burning can even favour the formation of carbon stars, increasing the surface $\mathrm{C} / \mathrm{O}$ through significant destruction of oxygen (and not through dredgeup of carbon). We can notice that the predicted luminosity range of the brightest carbon stars is larger for higher $M, \alpha$, and lower $Z$, and in most cases extends above the AGB termination luminosity as a consequence of the over-luminosity effect produced by envelope burning.

Acknowledgments. The author acknowledges the IAU for travel funding.

\section{References}

Baud B., Habing H.J., 1983, A\&A 127, 73

Blöcker T., Schönberner D., 1991, A\&A 244, L43 (BS91)

Blöcker T., 1995, A\&A 297, 727 (B95)

Boothroyd A.I., Sackmann I.-J., 1988, ApJ 328, 671

Costa E., Frogel J.A., 1996, AJ 112, 2607

Frost C.A., Cannon R.C., Lattanzio J.C., et al., 1998, A\&A 332, L17

Groenewegen M.A.T., de Jong T., 1993, A\&A 267, 410

Marigo P., 1998a, PhD Thesis, University of Padova

Marigo P., 1998b, A\&A, in press

Marigo P., Bressan A., Chiosi C., 1996, A\&A 313, 545

Marigo P., Bressan A., Chiosi C., 1998a, A\&A 331, 564

Marigo P., Girardi L., Bressan A., 1998b, A\&A submitted

Rebeirot E., Azzopardi M., Westerlund B.E., 1993, A\&AS 97, 603

Renzini A., Voli M., 1981, A\&A 94, 175

Vassiliadis E., Wood P.R., 1993, ApJ 413, 641

Wagenhuber J., Groenewegen M.A.T., 1998, A\&A 340, 183

Wood P.R., 1981, in Physical Processes in Red Giants, I. Iben Jr. and A. Renzini (eds.), D. Reidel Publishing Co., Dordrecht, p. 135 